\title{
Structural stability of nitrogen-doped ultrathin single-walled boron nanotubes: an ab initio study
}

\author{
Sandeep Kumar Jain • Pankaj Srivastava
}

Received: 26 November 2011 / Accepted: 6 March 2012/Published online: 23 March 2012

(c) The Author(s) 2012. This article is published with open access at Springerlink.com

\begin{abstract}
Ab initio calculations have been performed for determining structural stabilities of nitrogen-doped ultrathin single-walled boron nanotube. We have considered ultrathin boron nanotubes of diameters $<0.5 \mathrm{~nm}$, which include mainly three conformations of BNTs viz. zigzag $(5,0)$, armchair $(3,3)$ and chiral $(4,2)$ with diameters 4.60 , 4.78 and $4.87 \AA$, respectively. It has been investigated that $\alpha$-BNTs are highly stable, while hexagonal BNTs are found to be least stable. In view of increasing structural stability of hexagonal BNTs, substitutional doping of foreign atoms, i.e. nitrogen is chosen. The nitrogen atoms substitute the host atoms at the middle of the tubes. The substitution doping is made with all the three conformations. The structural stabilities of BNTs have been investigated by using density functional theory (DFT). Subsequently, the cohesive energy is calculated, which directly measures the structural stability. The cohesive energy of BNTs has been calculated for different nitrogen concentrations. We found that the structures get energetically more stable with increasing nitrogen concentration. Moreover, it is also revealed that all the three BNTs are almost equally stable for single-atom doping, while the armchair BNT $(3,3)$ is highly stable followed by zigzag $(5,0)$ and chiral $(4,2)$ BNTs for two- and three-atom doping. The structural stability is an important factor for realization of any physical device. Thus, these BNTs can be used for field emission, semiconducting and highly conducting devices at nanoscale.
\end{abstract}

S. K. Jain $(\bowtie)$. P. Srivastava

Computational Nanoscience and Technology Laboratory

(CNTL), ABV-Indian Institute of Information Technology

and Management, Gwalior 474015, India

e-mail: sandeepjain@iiitm.ac.in
Keywords Boron nanotube - Nitrogen-doped nanotube · Structural stability - Cohesive energy .

Ultrathin nanotubes · Formation energy

\section{Introduction}

Carbon nanotubes (Iijima 1991) have peculiar physical and chemical properties. It is found that carbon nanotubes (CNTs) are metallic as well as semiconducting, depending on the diameter and chirality (Dresselhaus et al. 1996). However, the electronic properties of boron-based nanotubes do not depend on diameter and chirality. The boron nitride nanotubes (BNNTs) are found to be semiconducting with large band gap (Rubio et al. 1994; Blasé et al. 1994) independent of diameter and chirality. The intriguing properties of nanotubes have fascinated the vast community of researchers and urged to explore nanotubes of other materials. Therefore, another nanotube of different material, i.e. Boron, was predicted (Boustani 1997a, b; Jain and Srivastava 2011) and succeedingly synthesized (Ciuparu et al. 2004) which verified the existence of BNTs. Thus, boron nanotubes (BNTs) are found to be metallic and independent of diameter and chirality. Moreover, there have been a lot of morphologies of BNTs such as hexagonal, triangular and $\alpha$-BNTs in contrast to only one morphology of CNTs, i.e. hexagonal. Boron sheets of various morphologies, viz. hexagonal, triangular and $\alpha$-boron sheet have been predicted based on ab initio calculations. Further, it has been found that $\alpha$-boron sheet is highly stable, while hexagonal boron sheet is least stable among these conformations. However, a recent study reveals that thinnest CNT-like BNTs are stable as predicted (Zhang et al. 2006) which instigated us to study hexagonal BNTs. 
The Aufbau principle was proposed for elemental boron (Boustani 1997a, b), which states that a stable boron cluster can be constructed from two basic units only: a pentagonal pyramidal $\mathrm{B}_{6}$ unit and a hexagonal pyramidal $\mathrm{B}_{7}$ unit. Further, the stable BNTs and BS can be constructed from only one unit, i.e. hexagonal pyramidal $\mathrm{B}_{7}$. Further, it is implied that quasiplanar (Boustani 1997a, b), tubular (Boustani and Quandt 1997; Gindulyte et al. 1998), convex and spherical (Boustani 1997a, b) boron clusters are made from these units. The existence of quasiplanar clusters or "sheet" was recently confirmed by experiment (Zhai et al. 2003), in perfect agreement with earlier theoretical predictions (Boustani et al. 1999). Moreover, the existence of quasiplanar boron cluster implies that boron nanotubes and boron fullerenes are formed because, during synthesis, a growing (quasi-) planar boron cluster tends to remove dangling bonds by forming closed tubular or polyhedral modifications. Beyond the Aufbau principle, we deal with CNT-like BNTs, made from hexagonal boron sheet. However, this has been found to be less stable.

The chemical doping of BNTs is a possible route towards controllable modification of electronic properties and enhancing stability. Doping may be implemented by intercalating foreign atoms into the open space or by substituting the host atoms with impurities. We have opted for substitutional doping with nitrogen, as it has a size almost equal to the boron atom. When nitrogen is doped in the CNTs, the electronic conductivity substantially increases. The enhancement of the density of states (DOS) at the Fermi level after substitution of nitrogen atoms into CNT increases the conductivity (Zhang et al. 2002, Kresse and Joubert 1999, Park et al. 2008). The nitrogen-doped SWCNTs (Min et al. 2007) have been synthesized directly on $\mathrm{SiO}_{2} / \mathrm{Si}$ substrate at $450{ }^{\circ} \mathrm{C}$ by water plasma chemical vapour deposition. The synthesized nitrogen-doped CNTs have diameter in the range of 0.8-1.6 nm. Moreover, as the concentration of nitrogen atoms increases, the imperfection gradually increases. It is also investigated that $\mathrm{N}$-doped CNTs are less stable under electron irradiation than pristine CNTs. The dopant nitrogen atom can be displaced more easily than host carbon atoms (Loponen et al. 2006). The morphology of the original nanotube (Morant et al. 2008) is maintained after nitrogen bombardment except for the incorporation of pearl-shaped decoration. When CNTs are bombarded with $\mathrm{N}$ ions for short times, the nitrogen is mainly incorporated into the CNTs rather than the substrate. However, at a longer bombarding time, the incorporation of nitrogen into the substrate is also evident.

Carbon nanotubes of small diameters, $4 \AA$, have been investigated theoretically as well as experimentally (Machon et al. 2002; Yang et al. 2003; Liu and Chan 2002) and found that semiconducting zigzag $(5,0)$ CNT becomes metallic and chiral $(4,2)$. CNT remains semiconducting, but with modified band gaps. This transformation is caused by curvature effect. Conversely, CNT-like BNTs are found to be metallic, except for chiral $(4,2)$ BNTs which show semiconducting nature. We have also considered the same conformations of BNTs, i.e. armchair $(3,3)$, Zigzag $(5,0)$ and chiral $(4,2)$.

\section{Computational methodology}

We have performed pseudo potential plane wave calculations for determining the structural stability of small diameter BNTs. The considered conformations of BNTs are of three types, viz. armchair $(3,3)$, zigzag $(5,0)$ and chiral $(4,2)$, consisting of 12,20 and 56 atoms, respectively. These tubes are periodic along the $\mathrm{z}$-axis. The ball and stick models are presented in Fig. 1, in which OA and $\mathrm{OB}$ represent the cross section of the tube, while $\mathrm{OC}$ is along the axis of the tube. The diameters of these tubes are $4.60,4.78$ and $4.87 \AA$ for $(5,0),(3,3)$ and $(4,2)$ BNTs, respectively. The geometry structures of BNTs are optimized and the total energy of the ground state is calculated by DFT-based CASTEP (Clark et al. 2005) simulation tool. The plane wave cutoff energy is set to be $320 \mathrm{eV}$ and exchange correlation effects are described by generalized gradient approximation (GGA) (Perdew et al. 1996) proposed by Perdew-Burke-Ernzehrof (PBE).The integration is performed in the first brillouin zone (Monkhorst and Pack 1976) by using the k-points generated by $1 \times 1 \times 8$ grid parameters. The ultrasoft pseudopotential is used in the reciprocal space (Vanderbilt 1990). The geometry is optimized until the force on each atom becomes $<0.03 \mathrm{eV} /$ $\AA$. The intertubular distance is kept fixed at $10 \AA$ in order to avoid periodic image interaction. The bond length $\mathrm{B}-\mathrm{B}$ is chosen to be $1.67 \AA$. In addition, non-spin-polarized calculations are performed.

The cohesive energy $E_{\text {coh }}$ of the SWBNT was obtained from the total energy $E_{\text {tot }}$, the energy of a single free boron atom $\left(E_{1}\right)$ and the energy of the nitrogen atom $\left(E_{2}\right)$ using the following relation:

$E_{\mathrm{coh}}=\frac{\left(E_{\mathrm{tot}}-x E_{1}-y E_{2}\right)}{x+y}$

where $x$ and $y$ are the numbers of boron and nitrogen atoms in the supercell, respectively.

\section{Results and discussion}

\section{Structural stability}

We have carried out ab initio calculations for determining the structural stability of BNTs. In this study, we have 

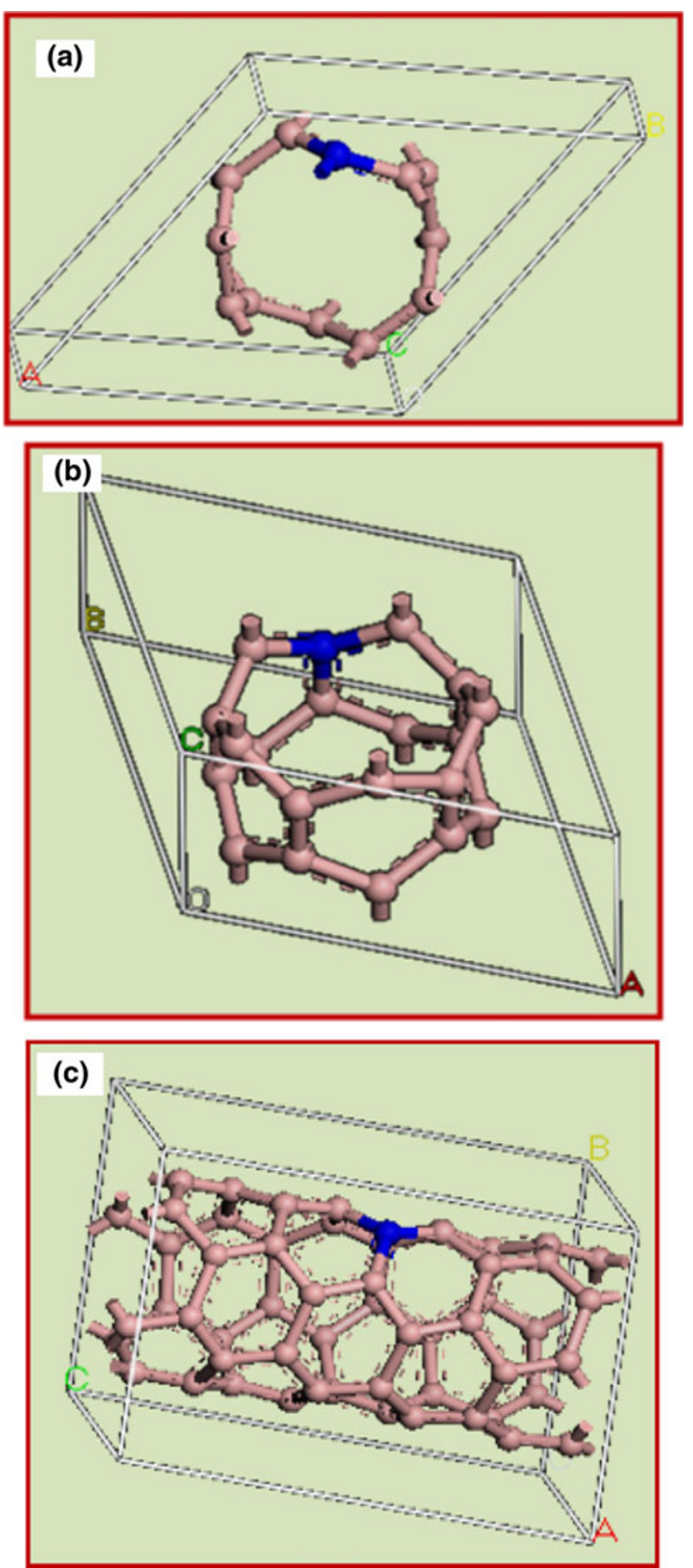

Fig. 1 The ball and stick model of ultrathin boron nanotubes for (a) armchair $(3,3)$, b zigzag $(5,0)$ and c chiral $(4,2)$ BNTs. The blue and brown colour atoms are of nitrogen and boron atoms, respectively

considered all the three conformations of BNTs, i.e. armchair $(3,3)$, zigzag $(5,0)$ and chiral $(4,2)$. The geometry of these BNTs is optimized and then total energy in the ground state is calculated. Subsequently, the cohesive energy is calculated by using total energy of the ground state of these BNTs. The total energy and cohesive energy
Table 1 Variation of total energy and cohesive energy of BNTs for different concentration of nitrogen

\begin{tabular}{lll}
\hline Nanotubes & $\begin{array}{l}\text { Total energy } \\
(\mathrm{eV})\end{array}$ & $\begin{array}{l}\text { Cohesive energy } \\
(\mathrm{eV})\end{array}$ \\
\hline Pristine armchair (3,3) & -912.83 & 5.57 \\
One-nitrogen atom doping & $-1,112.24$ & 6.18 \\
Two-nitrogen atom doping & $-1,307.30$ & 6.41 \\
Three-nitrogen atom doping & $-1,503.20$ & 6.73 \\
Pristine zigzag (5,0) & $-1,519.35$ & 5.47 \\
One-nitrogen atom doping & $-1,725.58$ & 6.18 \\
Two-nitrogen atom doping & $-1,921.12$ & 6.35 \\
Three-nitrogen atom doping & $-2,117.63$ & 6.56 \\
Pristine chiral (4,2) & $-4,289.37$ & 6.10 \\
One-nitrogen atom doping & $-4,485.15$ & 6.17 \\
Two-nitrogen atom doping & $-4,680.13$ & 6.22 \\
Three-nitrogen atom doping & $-4,877.57$ & 6.31 \\
\hline
\end{tabular}

of these BNTs are listed in Table 1. It is explicit from the table that the cohesive energy is found to be $6.18 \mathrm{eV}$ for single nitrogen atom doping. Moreover, this energy is independent of the diameter and chirality. Therefore, it can be interpreted that all the three conformations of BNTs, viz. armchair $(3,3)$, zigzag $(5,0)$ and chiral $(4,2)$, are found to be equally stable. Further, as the concentration of nitrogen increases to two atoms, the cohesive energy is further increased. Thus, this increment in cohesive energy implies increased stability.

The structural stability increases with the concentration of nitrogen. It is noteworthy that the cohesive energy is increased to a great extent in comparison to pristine BNTs. However, the structural stability of CNTs gets reduced after nitrogen doping. It was investigated in our earlier work (Jain and Srivastava 2011) that pristine chiral $(4,2)$ BNT was highly stable, followed by armchair $(3,3)$ and zigzag $(5,0)$ BNTs. This is also illustrated in the form of cohesive energy in Table 1, which confers the stability. When a single atom of boron is substituted by nitrogen, then the cohesive energy is found to be equal for all the three conformations, indicating that all the three BNTs are equally stable. Further, when the concentration of nitrogen is increased to two or three atoms, the cohesive energy does not remain the same for all the three BNTs, i.e. cohesive energy is different for different conformations. It is explicit in Fig. 2 that the cohesive energy of chiral BNTs $(4,2)$ is slightly altered for various concentrations of nitrogen. On the other hand, the cohesive energy of armchair and zigzag BNTs is increased to a large extent. For nitrogen-doped BNTs, it is found that armchair $(3,3)$ is highly stable, followed by zigzag $(5,0)$ and chiral $(4,2)$ BNTs for two- and three-atom doping, while for singleatom doping all the three BNTs are found to be equally stable. 


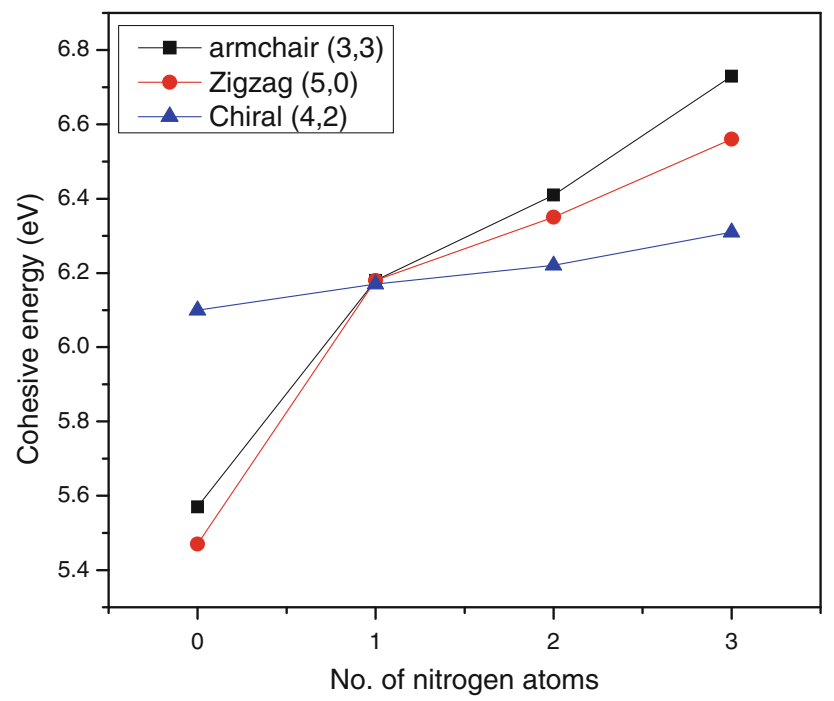

Fig. 2 The cohesive energies of all the three BNTs for different concentrations of nitrogen where black colour rectangles, red colour squares and blue colour triangles correspond to armchair $(3,3)$, zigzag $(5,0)$ and chiral $(4,2)$ BNTs respectively

Moreover, the formation energy of nitrogen-doped boron nanotube is calculated by using the ground state energy of relaxed structures. We determine the formation energy:

$E_{\text {formation }}=\frac{E_{\text {dopedBNT }}-E_{\text {pristine }}-x E_{\text {nitrogen }}}{n}$

where $E_{\text {dopedBNT }}$ is the total energy of our supercell with doped nitrogen atoms, $E_{\text {pristineBNT }}$ is the total energy of pristine BNT, $E_{\text {nitrogen }}$ is the total energy of an isolated nitrogen atom, $x$ is the total number of nitrogen atoms, and $n$ is the total number of atoms in the supercell of doped BNT.

The calculated values of formation energies $E_{\text {formation }}$ is shown in Fig. 3. The formation energy represents the favourability of the structures. Figure 3 depicts the formation energy of the considered conformations of BNTs with concentration of nitrogen impurity up to three atoms. It is revealed that the formation energy increases with the concentration of nitrogen impurity in all the three nanotubes. Therefore, nitrogen impurity makes these tubes more favourable, as well as stable. When a single atom of nitrogen substitutes host boron atom in all the three conformations of BNTs, the outcome is that the zigzag $(5,0)$ tube is highly favourable followed by armchair $(3,3)$ and chiral $(4,2)$. Single-atom-doped BNTs are almost equally stable as they have equal cohesive energies. However, the zigzag $(5,0)$ tube is most favourable, because this tube possesses the highest formation energy among the three conformations. Further, as the concentration of doping is increased, the formation energy is also increased. It is noteworthy here that zigzag $(5,0)$ BNTs have highly

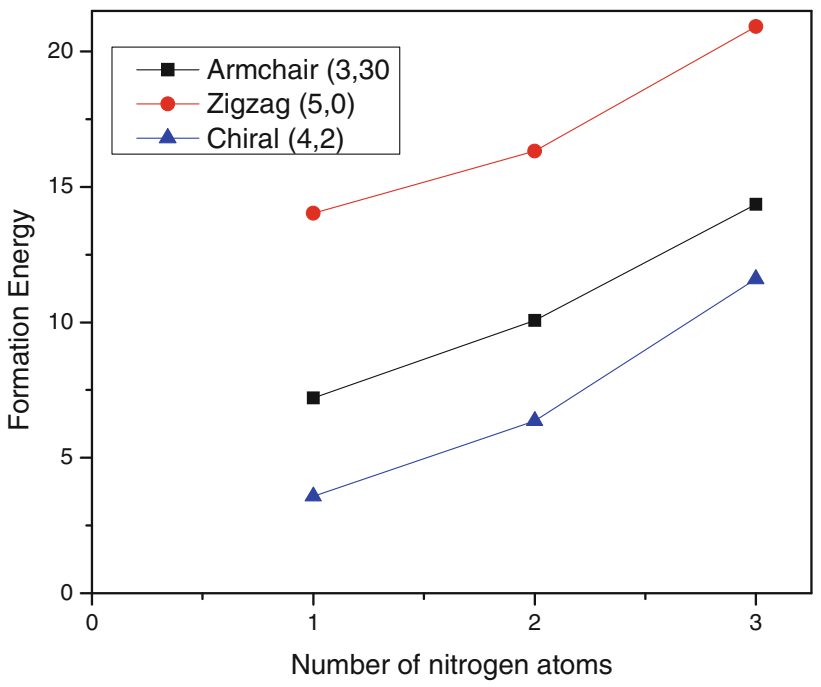

Fig. 3 The formation energies of all the three BNTs for different concentrations of nitrogen, where black colour rectangles, red colour spheres and blue colour triangles correspond to armchair $(3,3)$, zigzag $(5,0)$ and chiral $(4,2)$ BNTs respectively

favourable structures followed by armchair $(3,3)$ and chiral $(4,2)$ BNTs. Thus, cohesive energies and formation energies are in good agreement with each other. Both energies increase with nitrogen concentration.

\section{Conclusion}

The first principle calculations have been performed for determining the structural stabilities of ultrathin BNTs. It is revealed that armchair $(3,3)$ BNT is found to be highly stable, followed by zigzag $(5,0)$ and chiral $(4,2)$ BNTs for two- and three-atom doping, while for single-atom doping all the BNTs are found to be equally stable. We have performed DFT calculations to determine the ground state energy of all the three conformations of BNTs and subsequently calculated the cohesive energy. It is observed that the structures become energetically more stable after nitrogen doping. Unlike the CNTs, the structural stability of SWBNTs increases with the increase of nitrogen concentration. Thus, these tubes may be more suitable for field emission devices, touch screen devices and semiconducting devices.

Acknowledgments The authors are thankful to the Computational Nanoscience and Technology Lab (CNTL), ABV-Indian Institute of Information Technology and Management, Gwalior, for providing computational facility.

Open Access This article is distributed under the terms of the Creative Commons Attribution License which permits any use, distribution, and reproduction in any medium, provided the original author(s) and the source are credited. 


\section{References}

Blasé X, Robio A, Louie SG, Cohen ML (1994) Stability and band gap constancy of boron nitride nanotubes. Europhys Lett 28:335

Boustani I (1997a) New convex and spherical structures of bare boron clusters. J Solid State Chem 133:182-189

Boustani I (1997b) Systematic ab initio investigation of bare boron clusters: determination of the geometry and electronic structures of $B_{n}(n=2-14)$. Phys Rev B 55:16426-16438

Boustani I, Quandt A (1997) Nanotubes of bare boron clusters, ab initio and density functional study. Europhys Lett 39:527

Boustani I, Rubio A, Alonso JA (1999) Ab initio study of B7 clusters: competition between spherical, quasiplanar and tubular isomers. Chem Phys Lett 311:21-28

Ciuparu D, Klie RF, Zhu YM, Pfefferle L (2004) Synthesis of pure boron single wall nanotubes. J Phys Chem B 108:3967-3969

Clark SJ, Segall MD, Pickard CJ, Hasnip PJ, Probert MJ, Rafson K et al (2005) First principle methods using CASTEP. Z Kristollographie 220:567-570

Dresselhaus MS, Dresselhaus G, Eklund P (1996) Science of fullerenes and carbon nanotubes. Academic Press, San Diego

Gindulyte A, Lipscomb WN, Massa L (1998) Proposed boron nanotubes. Inorg Chem 37:6544

Iijima S (1991) Helical microtubules of graphitic carbon. Nature 354:56-58

Jain SK, Srivastava P (2011) Electronic and optical properties of ultrathin boron nanotubes - an ab initio study. Comp Mater Sci 50:3038-3042

Kresse G, Joubert D (1999) From ultrasoft pseudopotentials to the projector augmented-wave method. Phys Rev B 59:1758-1775

Liu HJ, Chan CT (2002) Properties of $4 \AA$ carbon nanotubes from first-principles calculations. Phys Rev B 66:115416-115420

Loponen T, Krasheninnikov AV et al. (2006) Nitrogen-doped carbon nanotubes under electron irradiation simulated with a tightbinding model. Phys Rev B 74:073409 (4 pp)
Machon M, Reich S, Thomsen C, Sanchez-Portal D, Ordejon P (2002) $\mathrm{Ab}$ initio calculations of the optical properties of $4 \AA$-diameter single walled nanotubes. Phys Rev B 66:155410-155415

Min YS, Bae EJ et al. (2007) Growth and characterization of nitrogen-doped single-walled carbon nanotubes by water-plasma chemical vapour deposition. Nanotechnology 18:285601(4 pp)

Monkhorst HJ, Pack JD (1976) Special points for brillouin-zone integrations. Phy Rev B 13:5188-5192

Morant C, Torres R, Jimenez I, Sanz JM, Elizalde E (2008) Characterization of nitrogen-doped carbon nanotubes by atomic force microscopy and X-ray absorption near edge spectroscopy. Nanosci Nanotechnol 8:1-6

Park H-L, S-C YI, Chung Y-C (2008) Electronic properties of nitrogen-doped carbon nanotubes with strain: ab initio method Approach. Jpn J Appl Phys 47:5062-5065

Perdew JP, Burke K, Ernzerhof M (1996) Generalized gradient approximation made simple. Phys Rev Lett 77:3865-3868

Rubio A, Corkill J, Cohen ML (1994) Theory of graphitic boron nitride nanotubes. Phys Rev B 49:5081-5084

Vanderbilt D (1990) Soft self-consistent pseudopotentials in a generalized eigenvalue formalism. Phys Rev B 41:7892-7895

Yang XP, Weng HM, Dong J (2003) Optical properties of 4 A singlewalled carbon nanotubes inside zeolite channels studied from first principles calculations. Euro Phys J B 32:345-350

Zhai HJ, Kiran B, Li J, Wang LS (2003) Planar-to-tubular structural transition in boron clusters: $\mathrm{B}_{20}$ as the embryo of single-walled boron nanotubes. Nat Mater 2:827

Zhang G, Duan WH, Gu BL (2002) Effect of substitutional atoms in the tip on field-emission properties of capped carbon nanotubes. Appl Phys Lett 80:2589 (3 pp)

Zhang D, Zhu R, Liu C (2006) Density functional theory study on the geometrical and electronic structures of a new thinnest boron nanotube. J Mater Chem 16:2429-2433 